\title{
TAFMEC Special Issue on Hydrogen Embrittlement of Metallic Materials: Fatigue and Fracture
}

Hydrogen Embrittlement is a phenomenon of great interest in the scientific community because of its undesirable effects on metallics materials. Loss of ductility, drastic reduction in fracture toughness, and fatigue resistance degradation are some of the most notorious effects of hydrogen in metals. A phenomenon of both of complex fundamental nature and great technological importance, hydrogen embrittlement is attracting an ever-increasing interest due to the higher susceptibility of modern high-strength alloys and the important role that hydrogen can play as an energy carrier of the future.

This special issue is focused on reporting the latest scientific progress on the study of hydrogen embrittlement across scales, from nano to macro, with special focus on the implications for fracture and fatigue properties. Among others, the topics covered in this Special Issue include the micromechanisms of hydrogen damage, the development of new computational methods for modelling hydrogen embrittlement, the influence of plasticity and strain gradient plasticity on diffusion and cracking, hydrogen assisted fatigue and fracture testing, and predictive modelling for engineering assessment.

Invitations to contribute were sent to 20 academics of renowned expertise in the field of hydrogen assisted cracking in May 2020. Despite the impact of a global pandemic that was then at its peak, the vast majority of those contacted enthusiastically accepted and later submitted original contributions of a very high quality, which showcase the rigour and the breadth of current research endeavours in the hydrogen embrittlement community. We are very grateful to these colleagues that, despite the circumstances, accepted our invitation to contribute to the special issue. These contributions are expected to have a notable impact on future academic research and also on industrial practice. We would also like to thank Prof. Luca Susmel, Theoretical and Applied Fracture Mechanics (TAFMEC) Editor-in-Chief for making this issue possible. All the papers underwent the regular strict review process of TAFMEC.

\author{
Guest Editors \\ Emilio Martínez-Pañeda \\ Imperial College London, UK \\ E-mail address: e.martinez-paneda@imperial.ac.uk
}

Jesus Manuel Alegre

University of Burgos, Spain

E-mail address: jalegre@ubu.es 